\title{
Mechanical Property Analysis of rubber-like materials under Large Deformation in Uniaxial Tension, Biaxial Tension and Expansion of Cylindrical Membrane
}

\author{
Jianbing SANG, Qi HAN, Yi ZHANG, Jingyuan WANG, Xiaolei LI \\ School of Mechanical Engineering, Hebei University of Technology, Tianjin, 300130, China, \\ E-mail: sangjianbing@126.com \\ crossref http:// http://dx.doi.org/10.5755/j01.mech.24.1.18377
}

\section{Introduction}

Rubber-like materials have been applied in a wide variety of different industrial areas, such as seals, vehicles tire, hydraulic hoses, shock and vibration absorbers and so on [1-2], which can undergo large deformation and exhibit large nonlinear elastic behaviour. The dominating mechanical behaviour of rubber like materials is its extreme deformation and almost full recovery in unloading. Being subjected to large deformations, rubber like materials demonstrates nonlinear features inherently. Therefore, the selection of strain-energy density function is a vital for theoretical research. Many attempts have been made to develop a theoretical stress-strain relation. Mooney [3] proposed a phenomenological model with two parameters based on the assumption of a linear relation between the stress and strain during simple shear deformation. In 1948, Rivlin put forward the strain energy function model to the isotropic hyperelastic materials [4]. Later, Treloar [5] published a model based on the statistical theory, the so-called neo-Hookean material model with only one material parameter. Mooney and neo-Hookean strain energy function have played an important role in the development of the nonlinear hyperelastic theory and its applications. In 1972, Ogden [6-7] proposed a strain energy function expressed in terms of principal stretches, which is probably the bestknown example for the principal stretch-based constitutive formulations consistent with the Valanis-Landel hypothesis.

In 1996 Alan Gent [8] proposed a new strain energy function for the non-linear elastic behaviour of rubber like materials. Because of its formal simplicity, this model has been widely applied large elastic deformations of solids [9-10]. The Gent model belongs to the class of the generalized neo-Hookean materials.

In 1997, according to the tensile and compression properties of rubber like materials, Gao proposed the following strain energy function [11].

$$
W=A\left(I_{1}^{n}+I_{-1}^{n}\right)
$$

In which, $A$ and $n$ are material parameters and $I_{-1}=I_{2} / I_{3} . I_{1}, I_{2}$ and $I_{3}$ are the three strain invariants. The calculation indicates that the greater the tensile strain, the larger the $I_{1}$; the greater the compressive strain, the larger the $I_{-1}$. The two complement each other, which can describe the finite deformation features of the materials.

From Eq. (2), we can see that strain energy function can't meet the conditions of strain energy function $W=0$ apparently when $I_{1}=I_{2}=3$ and $I_{3}=1$. In other words, it should meet there is no strain energy function at initial spontaneous configuration. For the incompressible material, when $n=1$, the expression Eq. (2) can't be simplified to Neo-Hookean material, nor to Mooney-Rivlin material.

Based on Gao's constitutive model, A modified strain energy function for the incompressible rubber like materials has been proposed by Sang [12] as:

$$
W=A\left[\left(I_{1}^{n}-3^{n}\right)+\alpha\left(I_{2}^{n}-3^{n}\right)\right],
$$

where: $\alpha \quad(0 \leq \alpha \leq 1)$ is the material parameter reflecting $I_{2}$ 's influence on stress distribution. From the new constitutive model Eq. (2), it can be seen that when $n=1$ and $\alpha=0$, the modified strain energy function can be transformed to Neo-Hookean model; when $n=1$, it can be transformed to Mooney-Rivlin model. Based on strain energy function Eq. (2), the expression of Cuachy stress tensor can be achieved as follow:

$$
\sigma=-p I+2 \frac{\partial W}{\partial I_{1}} B-\frac{\partial W}{\partial I_{2}} B^{-1}
$$

where: $I$ is unit tensor. $p$ is the undetermined scalar function that justifies the incompressible internal constraint conditions.

\section{Analysis of uniaxial tension of rubber like materials}

For uniaxial tension of rubber like materials, we take $\lambda_{2}=\lambda_{3}$. For incompressibility, the following expression can be achieved as:

$$
\lambda_{1}=\lambda, \lambda_{2}=\lambda_{3}=\lambda^{-1 / 2}
$$

Based on the modified strain energy function, the tension Cauchy stress can be expressed as:

$$
\sigma_{11}=2 A n\left[\left(\lambda^{2}-\lambda_{-1}\right)\left(\lambda^{2}+2 \lambda^{-1}\right)^{n-1}-\alpha\left(\lambda^{-2}-\lambda\right)\left(2 \lambda+\lambda^{-1}\right)^{n-1}\right] .
$$


Based on Eq. (5), the relations between Cauchy stress and principal stretch have been plotted with different material parameters. When the parameter $\alpha$ is given ( $\alpha=0.1$ ), as illustrated in Fig. 1. As the constitutive parameter $n$ increases, the Cauchy stress becomes much greater and it has the reinforcement feature apparently. The stress-strain curve becomes sharp lifting in large deformation when $n=1.6$. As is shown in Fig. 2, for the given $n=1.2$, the larger the $\alpha$ is, the greater the stress is, however, its influence is smaller than that of reinforcement parameter $n$.

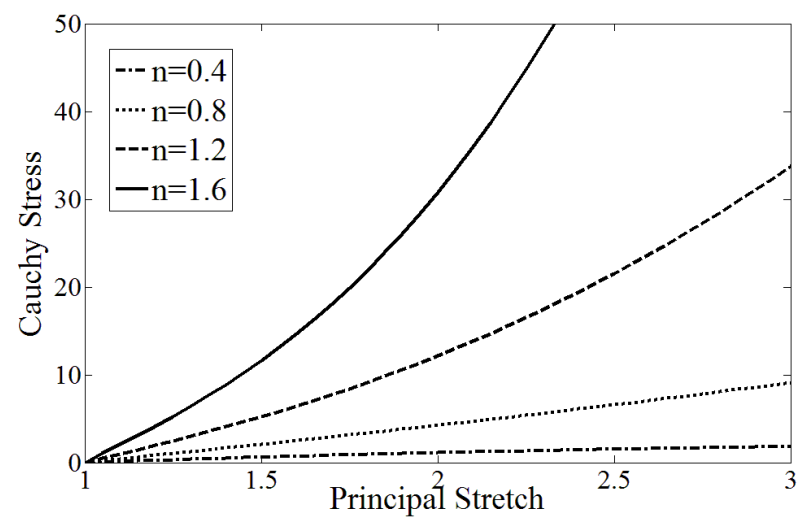

Fig. 1 Theoretical Solution of Cauchy stress versus principal stretch for different material parameter $n$ (uniaxial tension)

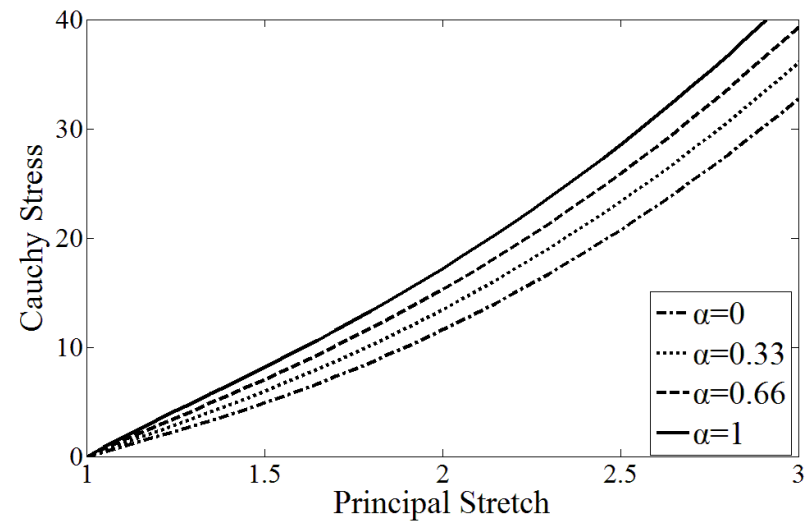

Fig. 2 Theoretical Solution of Cauchy stress versus principal stretch for different material parameter $\alpha$ (uniaxial tension)

According to the standard uniaxial specimen of rubber like materials, the narrow part of the standard specimen thickness is $2.0 \mathrm{~mm} \pm 0.2 \mathrm{~mm}$, and the length of it is $25.0 \mathrm{~mm} \pm 0.5 \mathrm{~mm}$. Based on the finite element software ABAQUS, three-dimensional finite element model of rubber specimen is set up. In the model, there are 1872 elements and 3081.nodes. Due to the incompressibility of the rubber material, the entity hybridizational element $\mathrm{C} 3 \mathrm{D} 8 \mathrm{H}$ was used and it analyzed in ABAQUS/Standard. A fixed displacement is applied to the nodes belonging to the left widest part of the specimen and the summation of reactions along the axial direction at nodes on the right widest part of the specimen gives the entity of the load. In order to implement the modified strain energy function Eq. (2) into the finite element procedure ABAQUS, non-linear finite element analysis was performed by a user subroutine when defining the material properties of rubber like materials, which allows the users to define the derivatives of the strain energy functions with respect to either the strain invariants or the principal stretches.

In order to compare the finite element results and theoretical results, the relation curves between Cauchy stress and principal stretch $\lambda$ have been also plotted with different material parameters as shown in Figs. 3 and 4. We can also see when the parameter $\alpha$ is given $(\alpha=0.1)$, as the constitutive parameter $n$ increases, the Cauchy stress becomes greater. Therefore, $n$ is considered as the material's reinforcement parameter. As is shown in Fig. 4, for the given $n=1.2$, the larger the $\alpha$ is, the greater the stress is, however, its influence is smaller than that of reinforcement parameter $n$. We can see the finite element results is excellent reasonable agreement with the theoretical results, which verify the theoretical analysis is reasonable.

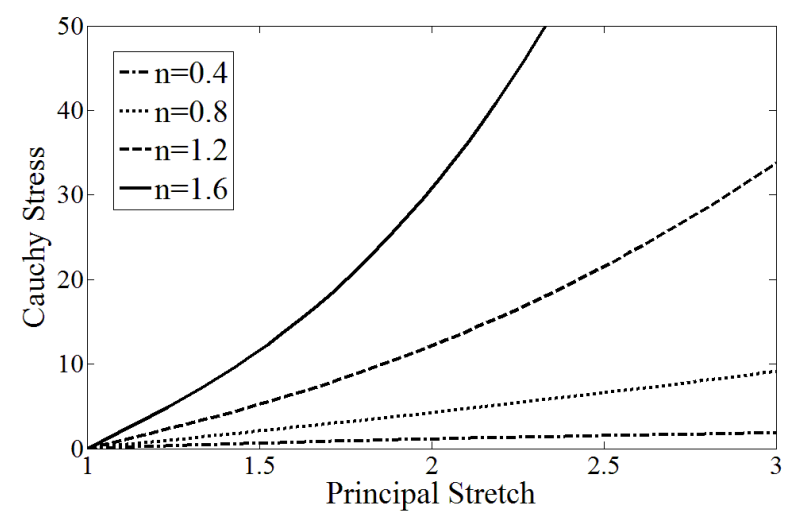

Fig. 3 Numerical Solution of Cauchy stress versus principal stretch for different material parameter $n$ (uniaxial tension)

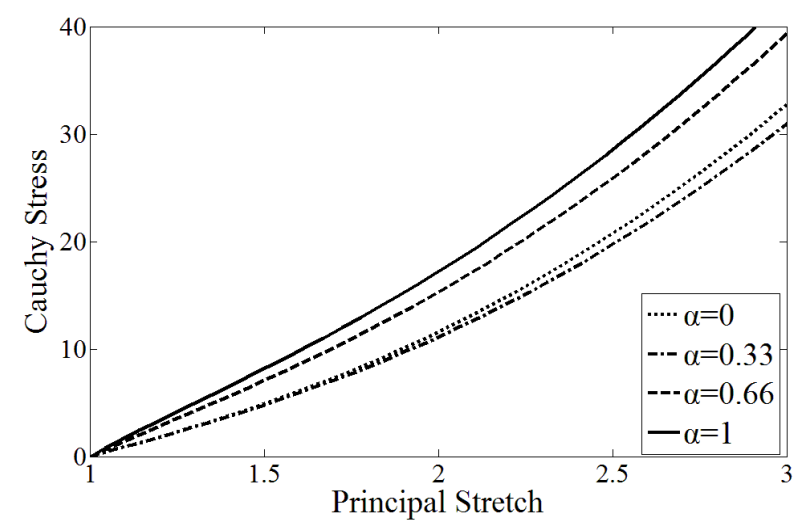

Fig. 4 Numerical Solution of Cauchy stress versus principal stretch for different material parameter $\alpha$ (uniaxial tension)

\section{Analysis of biaxial tension of rubber like materials}

For biaxial tension of rubber like materials, we take $\lambda_{2}=\lambda_{3}=\lambda$. For incompressibility, the following expression can be achieved as:

$$
\lambda_{2}=\lambda_{3}=\lambda, \quad \lambda_{1}=\lambda^{-2}
$$

Based on the modified strain energy function, the tension Cauchy stress can be expressed as: 


$$
\sigma_{22}=\sigma_{33}=\frac{2 A n}{\rho_{0}}\left[\left(\lambda^{-4}+2 \lambda^{2}\right)^{n-1}\left(\lambda^{2}+\lambda^{-4}\right)-\alpha\left(2 \lambda^{-2}+\lambda^{4}\right)^{n-1}\left(\lambda^{-2}+\lambda^{4}\right)\right] .
$$

For biaxial tension, the relations between Cauchy stress and principal stretch have been plotted with different material parameters as shown in Figs. 5 and 6. From Fig. 5 we can see the stiffness of rubber like materials increase as the increasing of material parameter $n$, which verify the material parameter $n$ can be considered as the material's reinforcement parameter. As shown in Fig. 6, for the given $\alpha=0$, the second principal strain invariant has no effect on the rubber like materials. The mechanical property of rubber like materials has been changed apparently as increasing of material parameter $\alpha$ which is not same as uniaxial tension.

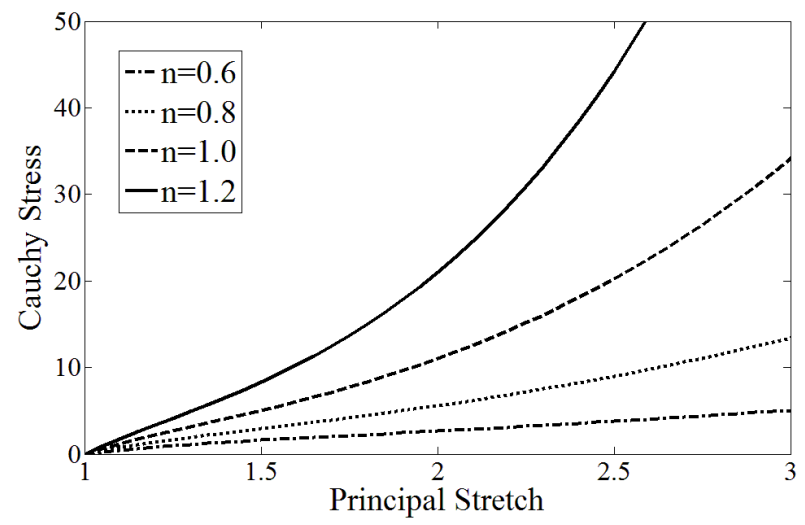

Fig. 5 Theoretical Solution of Cauchy stress versus principal stretch for different material parameter $n$ (biaxial tension)

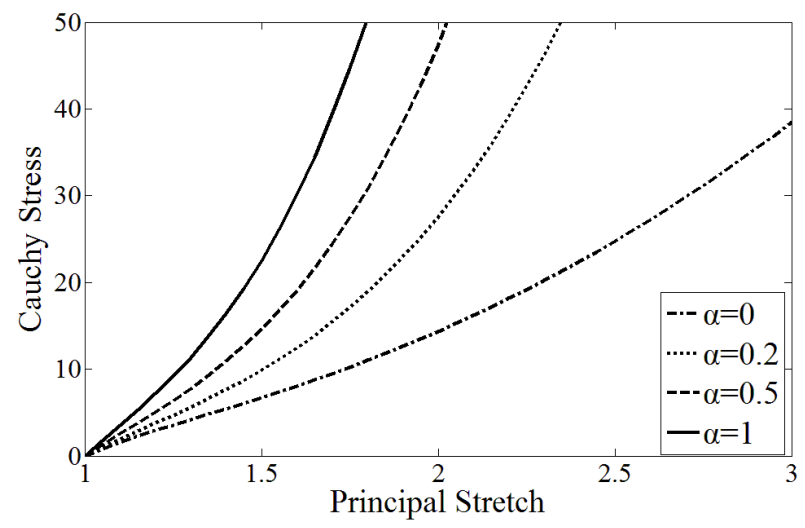

Fig. 6 Theoretical Solution of Cauchy stress versus principal stretch for different material parameter $\alpha$ (biaxial tension)

In order to in comparison with the theoretical results, the finite element analysis of biaxial rubber specimen has been proposed. The finite element model length is $100 \mathrm{~mm}$, and there are 5000 elements and 7803 nodes. Due to the incompressibility of the rubber material, the entity hybridization element C3D8H was also used and analyzed in ABAQUS / Standard. The rubber specimen was fixed at the left side and top side. On the right side and down side of the specimen gives the entity of the load. Non-linear finite element analysis was also performed by utilizing the user subroutine.
In order to compare the finite element results and theoretical results, the relation curves between Cauchy stress and principal stretch $\lambda$ have been also plotted with different material parameters as shown in Figs. 7 and 8 . We can also see when the parameter $\alpha$ is given ( $\alpha=0.1$ ), as the constitutive parameter $n$ increases, the Cauchy stress becomes greater. Therefore, $n$ is considered as the material's reinforcement parameter. For the given $n=1.2$, the larger the $\alpha$ is, the greater the stress is, however, its influence on the mechanical property of rubber like materials is apparently, which is not same as uniaxial tension. We can also see the finite element results are excellent reasonable agreement with the theoretical results, which verify the theoretical analysis is reasonable.

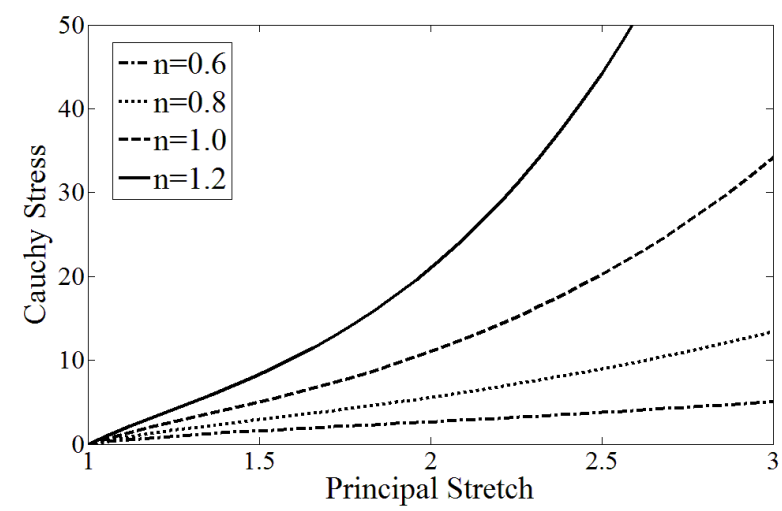

Fig. 7 Numerical Solution of Cauchy stress versus principal stretch for different material parameter $n$ (biaxial tension)

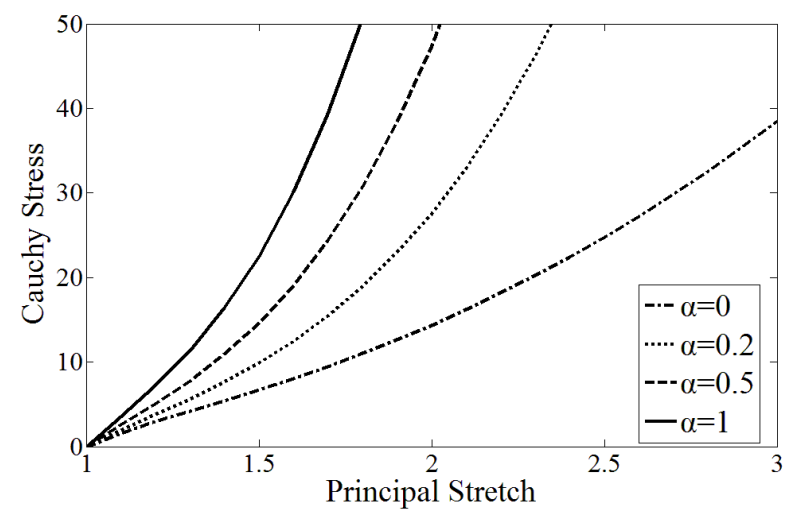

Fig. 8 Numerical Solution of Cauchy stress versus principal stretch for different material parameter $\alpha$ (biaxial tension)

\section{Analysis of cylindrical rubber membrane under internal pressure}

For cylindrical rubber membrane under uniform pressure, if $(R, \theta, Z)$ and $(r, \theta, z)$ are the coordinate of rubber membrane before deformation and after deformation respectively, then the deformation pattern of the rubber tube can be expressed as: 


$$
r=f(R), \theta=\Theta, Z=\lambda Z \text {. }
$$

Define $\lambda_{r}, \lambda_{\theta}$ and $\lambda_{z}$ are the principal stretch of radial direction, circumferential direction and axial direction of cylindrical membrane, which can be expressed as:

$$
\lambda_{r}=\frac{d r}{d R}=\left(\lambda \lambda_{z}\right)^{-1}, \lambda_{\theta}=\frac{r}{R}=\lambda, \lambda_{z}=\lambda_{z}
$$

The Cuachy-Green deformation tensor $B$ can be expressed as follows:

$$
[B]=[F][F]^{\mathrm{T}}=\left[\begin{array}{ccc}
\lambda_{r}^{2} & 0 & 0 \\
0 & \lambda_{\theta}^{2} & 0 \\
0 & 0 & \lambda_{z}^{2}
\end{array}\right]=\left[\begin{array}{ccc}
\left(\lambda \lambda_{z}\right)^{-2} & 0 & 0 \\
0 & \lambda^{2} & 0 \\
0 & 0 & \lambda_{z}^{2}
\end{array}\right] .
$$
shown as:

Substitute Eq. (10) into Eq. (3) and utilize the modified strain energy function Eq. (2), the Cauchy stress can be

$$
\left\{\begin{array}{l}
\sigma_{r r}=-p+2 \frac{\partial W}{\partial I_{1}} \lambda_{r}^{2}-2 \frac{\partial W}{\partial I_{2}} \lambda_{r}^{-2}=-p+2 A n I_{1}^{n-1} \lambda_{r}^{2}-2 A n \alpha I_{2}^{n-1} \lambda_{r}^{-2} \\
\sigma_{\theta \theta}=-p+2 \frac{\partial W}{\partial I_{1}} \lambda_{\theta}^{2}-2 \frac{\partial W}{\partial I_{2}} \lambda_{\theta}^{-2}=-p+2 A n I_{1}^{n-1} \lambda_{\theta}^{2}-2 A n \alpha I_{2}^{n-1} \lambda_{\theta}^{-2} \\
\sigma_{z z}=-p+2 \frac{\partial W}{\partial I_{1}} \lambda_{z}^{2}-2 \frac{\partial W}{\partial I_{2}} \lambda_{z}^{-2}=-p+2 A n I_{1}^{n-1} \lambda_{z}^{2}-2 A n \alpha I_{2}^{n-1} \lambda_{z}^{-2}
\end{array}\right.
$$

Based on the hypothesis of membrane $\sigma_{r r}=0$, we

$$
p=2 A n I_{1}^{n-1} \lambda_{r}^{2}-2 A n \alpha I_{2}^{n-1} \lambda_{r}^{-2} .
$$
can get:

The Eq. (11) can be transformed as:

$$
\left\{\begin{array}{l}
\sigma_{\theta \theta}=2 A n\left(\lambda_{\theta}^{2}-\lambda_{r}^{2}\right)\left(\lambda_{\theta}^{-2} \lambda_{z}^{-2}+\lambda_{\theta}^{2}+\lambda_{z}^{2}\right)^{n-1}+2 A n \alpha\left(\lambda_{r}^{-2}-\lambda_{\theta}^{-2}\right)\left(\lambda_{z}^{-2}+\lambda_{\theta}^{2} \lambda_{z}^{2}+\lambda_{\theta}^{-2}\right)^{n-1}, \\
\sigma_{z z}=2 A n\left(\lambda_{z}^{2}-\lambda_{r}^{2}\right)\left(\lambda_{\theta}^{-2} \lambda_{z}^{-2}+\lambda_{\theta}^{2}+\lambda_{z}^{2}\right)^{n-1}+2 A n \alpha\left(\lambda_{r}^{-2}-\lambda_{z}^{-2}\right)\left(\lambda_{z}^{-2}+\lambda_{\theta}^{2} \lambda_{z}^{2}+\lambda_{\theta}^{-2}\right)^{n-1} .
\end{array}\right.
$$

If $R$ and $r$ are the radius of cylindrical polymer membrane according to before deformation and after deformation. And $H$ and $h$ is the thickness of cylindrical rubber membrane according to before deformation and after deformation. We can get:

$$
h=\lambda_{r} H, r=\lambda_{\theta} R
$$

By utilizing the Eq. (15) and Eq. (13), we can get:

$$
A n\left(\lambda_{\theta}^{-2} \lambda_{z}^{-2}+\lambda_{\theta}^{2}+\lambda_{z}^{2}\right)^{n-1}\left[\left(\lambda_{\theta}^{2}-\lambda_{r}^{2}\right)-2\left(\lambda_{z}^{2}-\lambda_{r}^{2}\right)\right]+A n \alpha\left(\lambda_{\theta}^{2} \lambda_{z}^{2}+\lambda_{\theta}^{-2}+\lambda_{z}^{-2}\right)^{n-1}\left[\left(\lambda_{r}^{-2}-\lambda_{\theta}^{-2}\right)-2\left(\lambda_{r}^{-2}-\lambda_{z}^{-2}\right)\right]=0 .
$$

When constitutive parameter $\alpha=0$, from Eq. (16), we can get:

Substitute Eq. (17) into the first Eq. (18) and nondimensional pressure is introduced we can get:

$$
\begin{aligned}
& \left\{\begin{array}{l}
\lambda_{\theta}=\sqrt{\lambda_{z}^{2}+\frac{\sqrt{\lambda_{z}^{6}-1}}{\lambda_{z}}}, \\
\lambda_{z}=\sqrt{\frac{\lambda_{\theta}^{2}}{4}+\frac{\sqrt{\lambda_{\theta}^{6}+8}}{4 \lambda_{\theta}} .} \\
p^{\#}=\frac{1}{2} \frac{1}{\lambda_{z}\left(\lambda_{z}^{2}+\frac{\sqrt{\lambda_{z}^{6}-1}}{\lambda_{z}}\right)}\left[\lambda_{z}^{2}+\frac{\sqrt{\lambda_{z}^{6}-1}}{\lambda_{z}}-\frac{1}{\lambda_{z}^{2}\left(\lambda_{z}^{2}+\frac{\sqrt{\lambda_{z}^{6}-1}}{\lambda_{z}}\right)}\right]\left[\frac{1}{\lambda_{z}^{2}\left(\lambda_{z}^{2}+\frac{\sqrt{\lambda_{z}^{6}-1}}{\lambda_{z}}\right)}+2 \lambda_{z}^{2}+\frac{\sqrt{\lambda_{z}^{6}-1}}{\lambda_{z}}\right]^{n-1},
\end{array},\right.
\end{aligned}
$$


$p^{\#}=\frac{1}{2} n \frac{1}{\lambda_{\theta}^{2} \sqrt{\frac{\lambda_{\theta}^{2}}{4}+\frac{\sqrt{\lambda_{\theta}^{6}+8}}{4 \lambda_{\theta}}}}\left[\lambda_{\theta}^{2}-\frac{1}{\lambda_{\theta}^{2}\left(\frac{\lambda_{\theta}^{2}}{4}+\frac{\sqrt{\lambda_{\theta}^{6}+8}}{4 \lambda_{\theta}}\right)}\right]\left[\frac{1}{\lambda_{z}^{2}\left(\lambda_{z}^{2}+\frac{\sqrt{\lambda_{z}^{6}-1}}{\lambda_{z}}\right)}+2 \lambda_{z}^{2}+\frac{\sqrt{\lambda_{z}^{6}-1}}{\lambda_{z}}\right]^{n-1}$.

In which, $p^{\#}=\frac{p R}{4 A H}$.

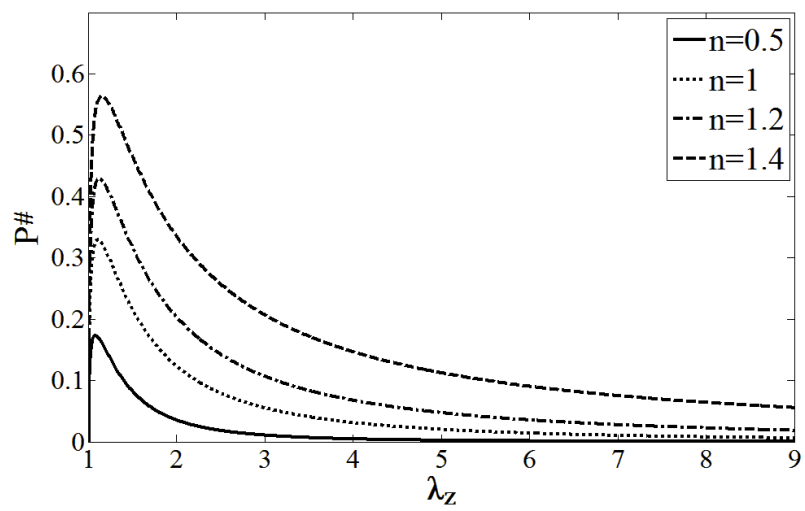

Fig. 9 The relation between $p^{\#}-\lambda_{z}(\alpha=0)$

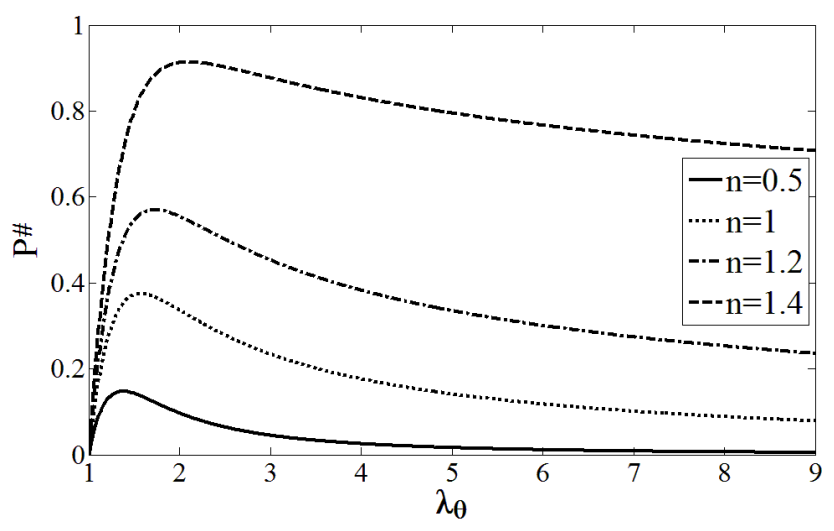

Fig. 10 The relation between $p^{\#}-\lambda_{\theta}(\alpha=0)$

In order to have a research on the cylindrical rubber membrane under internal pressure by the constitutive parameter $n$, the following circumstances is considered. When $\alpha$ is fixed, the distribution between internal pressure and circumferential principal stretch with the change of $n$ has been researched and the distribution between internal pressure and longitudinal principal stretch with the change of $n$ has also been researched. As shown in Fig. 9 and Fig. 10, for fixed material parameters $\alpha=0$, as the material parameter $n$ increases, circumferential and longitudinal principal stretch increases in accordance with internal pressure. The effect of constitutive parameter $n$ has a major impact on the mechanical properties of the cylindrical rubber membrane. When the material parameter $n$ takes higher value, the range of circumferential and longitudinal principal stretch is larger, which means the cylindrical rubber membrane has strong inflation capability and well elasticity. On the other hand, when the material parameter $\mathrm{n}$ takes lesser value, the range of circumferential and longitudinal principal stretch is smaller, which means rubber the inflation capability tube is weak. Especially instability of cylindrical rubber membrane will be occurring as the circumferential or longitudinal principal stretch increases, which means the stability analysis is necessary.

\section{Conclusion}

Based on the finite deformation theory, analysis of uniaxial tension and biaxial tension of rubber like materials has been proposed based on the constitutive model from Gao. In order to verify the correctness of theoretical results, the proposed strain energy function implemented in general-purpose FEA software. And then the nonlinear finite element procedure is presented for the analysis of rubber-like materials. From that we can get when the parameter $\alpha$ is given ( $\alpha=0.1)$, as the constitutive parameter $n$ increases, the Cauchy stress becomes greater. Therefore, $n$ is considered as the material's reinforcement parameter. For the given $n=1.2$, the larger the $\alpha$ is, the greater the stress is, however, its influence is smaller than that of reinforcement parameter $n$ for uniaxial tension and is apparently for biaxial tension, which mean the theoretical analysis is reasonable. Analysis of cylindrical rubber membrane has been proposed based on the proposed strain energy function. The results show that the constitutive parameter $n$ has a strengthening effect on the rubber membrane material and instability of cylindrical rubber membrane will be occur as the circumferential or longitudinal principal stretch increases.

\section{Acknowledgement}

This paper is supported by Tianjin Excellent Special correspondent Project (Grant No. 16JCTPJC53100) and Scientific Research Key Project of Hebei Province Education Department (Grand No. ZD2016083) and Natural Science Foundation of Hebei Province (Grant No. A2015202242; A2016202195).

\section{References}

1. Kwon, Y.D.; Kwon, S.B.; Lu, X.; Kwon, H. W. 2014. A Finite Element Procedure with Poisson Iteration Method Adopting Pattern Approach Technique for Near-Incompressible Rubber Problems, Advances in Mechanical Engineering 2014: 1-12. http://dx.doi.org/10.1155/2014/272574.

2. Lecte, A.S.; Verron, E.; Huneau, B. 2014. How to identify a hyperelastic constitutive equation for rubberlike materials with multiaxial tension-torsion experiments, Int. J Non Lin Mech 65: 260-270. http://dx.doi.org/10.1016/j.ijnonlinmec.2014.06.007.

3. Rivlin, R.S. 1948. Large elastic deformations of isotropic materials: I Fundamental concepts, II. Some uniqueness theorems for pure, homogeneous deformation, Philos Trans Roy Soc, Lond: Ser A 240: 459508 . 
http://dx.doi.org/ 10.1098/rsta.1948.0002; 10.1098/rsta.1948.0003.

4. Mooney, M.A. 1940. A theory of large elastic deformation, J Appl Phys 11: 582-592. http://dx.doi.org/10.1063/1.1712836.

5. Treloar, L.R.G. 1976. The mechanics of rubber elasticity, Proc Roy Soc, Lond: Ser A 351: 301-330. http://dx.doi.org/10.1002/polc.5070480110.

6. Ogden, R.W. 1972. Large deformation isotropic elasticity on the correlation of theory and experiment for the incompressible rubber-like solids. Proc Royal Soc, Lond: A. 326: 565-584. http://dx.doi.org/10.1098/rspa.1972.0026.

7. Ogden, R.W. 1972. Large deformation isotropic elasticity - on the correlation of theory and experiment for the compressible rubber-like solids. Proc Royal Soc, Lond: A. 328: 567-583. https://doi.org/10.1098/rspa.1972.0096.

8. Gent, A.S. 1996. A new constitutive ralation for rubber, Rubber Chem Technol. 69(1): 59-61. http://dx.doi.org/10.5254/1.3538357.

9. Mangan, R.; Destrade, M. 2015. Gent models for the inflation of spherical balloons, Int. J Nonlin Mech. 68: 52-58. http://dx.doi.org/10.1016/j.ijnonlinmec.2014.05.016.

10. Horgan, C.O. 2015. The remarkable Gent constitutive model for hyperelastic materials, Int. J Nonlin Mech. 68: $9-16$ http://dx.doi.org/10.1016/j.ijnonlinmec.2014.05.010.

11. Gao, Y.C. Large deformation field near a crack tip in a rubber-like material. Theor. Appl Frac Mech. 26: 155162. http://dx.doi.org/10.1016/S0167-8442(96)00044-4.

12.Sang, J.B.; Sun, L.F.; Xing, S.F. et al. 2014. Mechanical properties of polymer rubber materials based on a new constitutive model, Polym Polym Compos. 22(8): 693-698.
Jianbing Sang, Qi Han, Yi Zhang, Jingyuan Wang, Xiaolei Li

\section{MECHANICAL PROPERTY ANALYSIS OF RUBBER- LIKE MATERIALS UNDER LARGE DEFORMATION IN UNIAXIAL TENSION, BIAXIAL TENSION AND EXPANSION OF CYLINDRICAL MEMBRANE}

S u m m a r y

By utilizing the modified strain energy function from Gao, uniaxial tension and biaxial tension of rubber like materials has been researched. For verification purposes, the proposed constitutive model from Gao has been implemented in a general-purpose FEA software. And then the nonlinear finite element procedure is presented for the analysis of rubber-like materials. The volumetric incompressibility condition of the deformation from rubber like materials is included in the formulation by using the penalty method. By utilizing the FEA software ABAQUS, uniaxial tension and biaxial tension model from rubber like materials have been established. In order to compare the FEA results with theoretical results, curve stress-principal stretch has been plotted with different material parameters, which indicate the correctness and rationality of theoretical analysis. In the end, analysis of cylindrical rubber membrane has been proposed based on the proposed strain energy function. The results show that the constitutive parameter $\mathrm{n}$ has a strengthening effect on the rubber membrane material and instability of cylindrical rubber membrane will be occur as the circumferential or longitudinal principal stretch increases. This research has revealed the deformational mechanism and provided reasonable reference for the design of rubber like materials.

Key words: rubber like materials, uniaxial tension, biaxial tension, cylindrical membrane.

Received June 14, 2017

Accepted February 15, 2018 